\title{
Time for cancer Immunotherapy!
}

\author{
Volker Schirrmacher* \\ Head of Tumor-Immunology, Immunological and Oncological Center Cologne (IOZK), Hohenstaufenring, Germany
}

Research of immunology in the last 60 years had a fascinating and successful development. This is witnessed by many Nobel Prizes, e.g. for discoveries concerned with the genes and proteins that characterize the antigen-specific receptors of B- and T-lymphocytes. In both cases the high diversity and antigen specificity is generated from a restricted germline pool of variable and constant domain genes.

I described in "Quo vadis cancer therapy?" [1] in detail the progress in immunotherapy, oncolytic virotherapy and targeted therapies with small molecule inhibitors. The book is a plea for more immunotherapy, lower side effects and higher long-lasting efficacy.

The first clinically relevant success story is that of the development of monoclonal antibodies (mabs), which are products of B-lymphocytes. The first FDA-approved mab is trastuzumab (Herceptin). This targets the cell surface receptor HER2 expressed for instance by breast cancer cells. Meanwhile dozens of therapeutic mabs are available for application in patients with a large variety of cancer types.

The second clinically relevant success occured in recent years with the development of mabs targeting immune regulatory receptors on $\mathrm{T}$ cells, such as CTLA-4 or PD-1. These receptors deliver negative signals to activated $\mathrm{T}$ cells to stop their activity at the end of their antigenic response. Tumors often use this physiological regulatory mechanism for immune escape. They thereby shut-off anti-tumor reactivity coming from tumor-infiltrating T-cells. The clinical application of checkpoint inhibitory mabs, which interfere with this tumor immune escape mechanism, has resulted - in a proportion of melanoma and carcinoma cancer patients - in an improvement of long-term survival. Such results were received with great surprise and enthusiasm because this had never been achieved before, neither with cytostatic drugs nor with small molecule inhibitors.

Further milestones from immunology came from research on $\mathrm{T}$ cells and Dendritic cells. The MHC restriction of cytotoxic $\mathrm{T}$ lymphocytes (CTLs) was discovered in 1973, the genes coding for the antigen-specific T cell receptor in 1984 and the first human tumorassociated antigen (TAA) recognized by CTLs as a peptide-MHC complex was identified in 1991. The discovery of Toll-like receptors (TLRs) triggering innate immune reactivity and that of Dendritic cells functioning as professional antigen-presenting cells (APCs) for T-cells, were further milestones in the new millenium.

Immunotherapies based on T-cell immunity include active immunization with cancer vaccines and adoptive cellular therapies. Oncolytic viruses (OVs), also developed in the last 60 years, are very promising new biological agents. They have the capacity of tumor selective replication and of tumor toxicity (oncolysis). By inducing immunogenic tumor cell death, OVs also support the development of post-oncolytic anti-tumor immunity. Therefore, the combination of OVs with cancer vaccines [2] has its own logic.
Adoptive T-cell therapies involve for instance the transfer of allogeneic peripheral blood derived donor cells to achieve Graft-versusLeukemia effects in leukemia patients. They can also consist in the transfer of autologous immune T-cells. An especially promising variety is the transfer of reactivated patient-derived memory $\mathrm{T}$ cells from the bone marrow [3]. A new recent development involves modern gene transfer technologies. These allow to produce $\mathrm{T}$ cells with transfected TAA-specific T-cell receptors or with chimeric TAA-specific receptors (CARs) consisting of antibody binding sites fused to T-cell receptor signaling chains [4].

\section{Comparing immunotherapies with other cancer therapies}

Tolerance mechanisms within the immune system are important to understand the low side effects of immunotherapies.

During evolution of life on earth, the immune system gradually developed in multicellular organisms. It began with innate immunity mechanisms. Distinct pattern recognition receptors (PRRs) of innate immunity cells allow to sense different exogenous microbial molecules. PRRs thus distinguish foreign from self molecules so that the organism can defend itself against pathogens. Later, during vertebrate development, the adaptive immunity system evolved. This is characterized by lymphoid organs specialized for development of new cell types with a broad variety of cell surface receptors for antigen recognition. Special recombinase enzymes were developed to generate from a set of germline genes during somatic B- and T-lymphocyte maturation billiards of antigen-specific receptor specificities. Before mature lymphocytes equipped with such receptors are allowed to leave their respective primary lymphoid organ (bone marrow for B-cells and thymus for T-cells), they are selected against reactivity towards normal self antigens of the organism.

The term Horror autotoxicus was introduced by Paul Ehrlich at the beginnings of the $20^{\text {th }}$ century to describe that the humoral immune system (based on B-cells) has developed mechanisms to avoid self destruction. In the 1950s, Sir Macfarlane Burnet described a cellular mechanism (based on T-cells) to prevent auto-immune reactions and introduced for this the term self tolerance. Meanwhile we distinguish between central and peripheral tolerance mechanisms. The late Bruno Kyewski and colleagues elucidated the sophisticated mechanism of central thymic self tolerance and its role in preventing organ-specific autoimmunity [5]. Markus Feuerer and colleagues recently described tissue-restricted regulatory $\mathrm{T}$ (Treg) cells that integrate multiple waves

*Correspondence to: Volker Schirrmacher, Head of Tumor-Immunology, Immunological and Oncological Center Cologne (IOZK), Hohenstaufenring 3032, D-50674 Cologne (Köln), Germany, Tel: +49 (0)221/ 420399 25; E-mail: V.Schirrmacher@web.de

Received: July 02, 2018; Accepted: July 12, 2018; Published: July 14, 2018 
of epigenetic reprogramming. They help to maintain self-tolerance and organ homeostasis [6].

When comparing immunotherapy with other therapies there is another characteristic difference, namely that of a memory function. Immunological memory serves the purpose of obtaining longterm protection and long-term health effects. The details of the immunological memory function are not yet entirely understood but it is likely that they are connected to special niches in the bone marrow and to a state of regulation that is reminiscent to that of stem cells.

Neither radiotherapy nor chemotherapy have a memory function. When the cancer is not entirely destroyed, the remaining cancer cells, in particular the cancer stem cells, can develop new growths.

When we analyzed the repertoire in breast cancer patients of bone marrow derived memory T-cells against TAAs, each patient showed its own pattern of reactivity [7]. There was a multitude of target molecules that were recognized in individual patients. This polyspecific reactivity did not cause any problem with side effects. The individuality of the immune memory repertoire seems to mimic the individuality of the cancers that arise in patients.

Another relevant question concerning future strategies of cancer treatment relates to that of targeting one or multiple molecules, molecule domains or molecule epitopes. This question is connected to that of development of cancer resistance mechanisms. Knowing that cancer is characterized by high heterogeneity and variability and can develop resistancies even to targeted therapies, the question is how to cope with this situation. One idea was to target a molecule such as a protein tyrosine kinase not just by one but by two inhibitors. Unfortunately, a protein tyrosine kinase inhibitor has side effects not much different from a cytostatic drug. Let us assume that there are six types of side effect per inhibitor. The addition of two inhibitors may reduce the likelihood of resistance development from the side of the cancer but the cancer patient is exposed to $2 \times 6$ side effects. Nobody can predict in which way the side effects will develop in an individual patient. There is a risk that side effects can potentiate each other. At the end, the situation may not be much better than with cytostatic drugs.

The situation is quite different with immunotherapy. Thanks' to the invention of immunological tolerance to self-tissues, immune T-cells reacting towards TAAs produce only low-level side effects, no matter whether they target only one or a multitude of molecules. Only when interfering with immune regulatory mechanisms there is the risk of severe side effects including autoimmune reactivity.

\section{Trends towards personalized and individualized therapies}

We observe at present a development towards personalized medicine. In medical oncology, this means individual tumor typing by procedures such as genomics, proteomics or pharmacogenomics and then adjusting the currently available drugs to the derived patterns of signaling pathways. In this way the drugs can be better targeted to subsets of patients.

The concept of individualized therapies based on immunotherapy is different. Each patient receives its own drug, for instance in form of a patient-derived autologous anti-cancer vaccine.

The rationale of individualized immunotherapy is multifold: Apart from the individuality of mutation-derived TAAs and the individuality of the cancer-reactive T-cell memory repertoire, their exists the individuality of MHC molecules and the phenomenon of MHC restriction of TAA recognition by T-cells.
There is a great potential in combining immunotherapy with treatment by oncolytic viruses (OVs). Oncolytic Virus Therapy is an emerging biological cancer treatment modality which uses replicationcompetent viruses to destroy cancer cells. OVs replicate selectively in cancer cells and damage cancerous tissue without causing harm to normal tissue.

One example of an OV is Newcastle Disease Virus (NDV). Oncolytic NDV has been applied to cancer patients already for more than 50 years and is very well tolerated [8]. In addition to its oncolytic potential, it was shown to be able to break T-cell tumor tolerance. It is also able to exert oncolytic activity against hypoxic cancer cells and to induce immunogenic cell death. In addition, NDV targets the Rac1 protein which is important for glioblastoma migration and invasion. Taken together, NDV is a biological agent with potential to break therapy resistance [9].

In Cologne, Germany, IOZK has developed a new strategy of cancer immunotherapy combining hyperthermia/oncolytic NDV pretreatment with specific autologous anti-tumor vaccination [2]. Results obtained at IOZK from treatment of glioblastoma multiforme (GBM) patients are similar to those recently published from Duke University Medical Center, Durham, USA. There they treated 61 recurrent GBM patients by intratumoral delivery of a recombinant nonpathogenic polio-rhinovirus chimera [10]. Importantly, overall survival, in contrast to historical controls, reached a plateau at about $20 \%$ at 2 years which sustained above 3 years.

\section{Promising future directions}

The approval by the FDA in recent years of targeted therapies with small molecule inhibitors and with monoclonal antibodies indicates that we are in a process in which cancer therapy is changing. Nevertheless, biological cancer therapies, like immunotherapy and oncolytic virus therapy are not yet part of standard therapies.

The success of Checkpoint inhibitors in clinical studies points to the importance of tumor immune escape mechanisms. What could be potential new targets of immunotherapy to avoid tumor immune escape mechanisms?

We know that cancers depend on a support system such as the tumor microenvironment with its stromal network. Interrupting this support system may be as efficient as targeting the cancer cells themselves. This view represents a change of paradigm in comparison to standard therapy.

Of course, the tumor itself needs to be targeted as well, in particular the cancer stem cells. Standard therapies like chemo- and radiotherapy target the cancer itself and try to reduce the number of dividing cancer cells. Eventually, the cancer will not only re-grow but it may have developed resistance mechanisms. The latter may occur through epigenetic mechanisms such as promoter DNA hypermethylation. This is a frequent phenomenon that can cause disruption of gene function.

Cancer stem cells can be targeted by oncolytic virus therapy. OVs have the potential to grow in and destroy even non-dividing cancer cells such as cancer stem cells. A strategy to target epigenetic mechanisms of cancer is the use of drugs like 5'-aza-cytidine. Targeting cancer stem cells and epigenetic mechanisms in the cancer together with activating the immune system towards TAAs should be a very promising concept for the future.

Our ability to predict what will be happening in the future with regard to cancer treatment is, of course, limited. Science is a very innovative discipline and many new developments may happen. 
Nevertheless, I predict the following areas of research as particularly promising : Epigenetics, the Tumor microenvironment, and the Immune system.

\section{References}

1. Schirrmacher V (2017) Quo vadis cancer therapy. Fascinating discoveries of the last 60 years. Lambert Academic Publishing, pp. 1-353.

2. Schirrmacher V, Lorenzen D, Van Gool SW, Stuecker W (2017) A new strategy of cancer immunotherapy combining hyperthermia/oncolytic virus pretreatment with specific autologous anti-tumor vaccination - A review. Austin Oncol Case Rep 2: 1006.

3. Feuerer M, Beckhove P, Bai L, Solomayer EF, Bastert G, et al. (2001) Therapy of human tumors in NOD/SCID mice with patient-derived reactivated memory T cells from bone marrow. Nat Med 7: 452-458. [Crossref]

4. Brown CE, Alizadeh D, Starr R, Weng L, Wagner JR, et al. (2016) Regression of glioblastoma after chimeric antigen-receptor therapy. N Engl J Med 375: 2561-2569.
5. Kyewski B, Klein L (2006) A central role for central tolerance. Annu Rev Immunol 24: 571-606.

6. Delacher M, Imbusch CD, Weichenhan D, Breiling A, Hotz-Wagenblatt A, et al. (2017) Genome-wide DNA-methylation landscape defines specialization of regulatory T cells in tissues. Nat Immunol 18:1160-1172.

7. Sommerfeldt N, Schütz F, Sohn C, Förster J, Schirrmacher V, et al. (2006) The shaping of a polyvalent and highly individual $\mathrm{T}$-cell repertoire in the bone marrow of breast cancer patients. Cancer Res 66: 8258-8265. [Crossref]

8. Schirrmacher V (2016) Fifty years of clinical application of Newcastle disease virus: Time to celebrate! Biomedicines $4: 3$.

9. Schirrmacher V (2015) Oncolytic Newcastle disease virus as a prospective anti-cancer therapy. A biological agent with potential to break therapy resistance. Expert Opin Biol Ther 15: 1757-71.

10. Desjardins A, Gromeier M, Herndon JE, Beaubier N, Bolognesi DP, et al. (2018) Recurrent Glioblastoma Treated with Recombinant Poliovirus. N Engl J Med. [Crossref]

Copyright: (C2018 Schirrmacher V. This is an open-access article distributed under the terms of the Creative Commons Attribution License, which permits unrestricted use, distribution, and reproduction in any medium, provided the original author and source are credited. 